\title{
TRADUCCIÓN Y DEMOCRACIA: LA NUEVA NARRATIVA ESPAÑOLA PUBLICADA EN ITALIA
}

\author{
Nuria PÉREZ VICENTE \\ Universidad de Trento, Urbino y Pavía / SELITEN@T \\ Nuria.Perez@lett.unitn.it
}

Resumen: El presente artículo se ocupa de las traducciones italianas, desde 1975 a 2000, de los «nuevos narradores», es decir, aquellos que publican su obra en un periodo fundamental de la historia española: el de la transición a la democracia. Trataremos de dar una visión panorámica sobre su situación en el mundo editorial italiano, llegando, debido entre otras cosas al aumento de publicaciones a partir de los años noventa, a conclusiones bastante favorables.

Résumé: Cet article parle des traductions italiennes des «nouveaux narrateurs» publiées entre 1975 et 2000, c'est à dire, ceux qui ont publié pendant le periode de la «Transition». L'article essaie de faire un tour d'horizont de leur situation chez les maisons d'édition italiennes. Grace au plus grand nombre d'oeuvres, qui ont été publiées dès les années '90, la situation de la littérature espagnole en Italie est assez favorable.

Palabras clave: Narrativa contemporánea española. Traducciones italianas. Transición. Democracia. 
Mots clé: Narrative contemporaine espagnole. Traductions italiennes. «Transition». Démocratie.

En el presente artículo ${ }^{1}$ queremos ocuparnos de las traducciones al italiano desde 1975 a $2000^{2}$ de los denominados «nuevos narradores», aquellos que publican su obra a partir de los años ochenta en concomitancia con un periodo fundamental de la historia española: el de la transición a la democracia. Aunque el margen de espacio disponible nos impida realizar un estudio pormenorizado de los volúmenes y analizar las traducciones, trataremos de dar una visión panorámica para llegar a unas conclusiones generales que nos orienten sobre la situación de nuestros autores en el mundo editorial italiano ${ }^{3}$.

El agotamiento del experimentalismo y los últimos latidos de la era de Franco anuncian la llegada de una nueva narrativa en España, desligada por fin de la triste herencia histórica y del compromiso social que había prevalecido hasta entonces. El relato comienza a ganar autonomía y un nutrido grupo de escritores de edad similar irrumpe en el escenario de las letras. La crítica italiana constata el inicio del nuevo periodo literario que se desencadena con la desaparición de la dictadura y, por encima de opiniones contrarias, parece muy bien dispuesta a aceptarlo. Coincide también en reconocer su heterogeneidad básica y alude a una sociedad que lejos de ofrecer res-

${ }^{1}$ Este estudio forma parte de la investigación llevada a cabo en nuestra tesis de doctorado europeo, defendida en enero de 2004 en Madrid y titulada Presencia de la narrativa española del siglo XX en Italia desde 1975: las traducciones. Se inserta en las actividades del Centro de Investigación de Semiótica Literaria Teatral y Nuevas Tecnologías (SELITEN@T) de la Universidad de Educación a Distancia (UNED), nacido en 1991 en el departamento de Literatura Española y Teoría de la Literatura de la Facultad de Filología de la misma universidad, dirigido por José Romera Castillo (director además de mi tesis de doctorado), y sigue la línea de trabajos precedentes realizados sobre Italia sobre la traducción de poesía y teatro españoles del siglo Xx (C. García Rodríguez, 2003a, 2003b). Para ampliar cualquier información concerniente a obras o autores, se puede consultar en la web del Centro, donde aparecerá próximamente en formato virtual. Se espera también su publicación en la editorial de la Universidad de Trento (Italia), colección «Laberinti». Sobre la presencia en Italia de las cuentistas españolas de los noventa vid. N. Pérez Vicente (2001); sobre novecentismo y vanguardismo, N. Pérez Vicente (2002); vid. N. Pérez Vicente (2003) para un panorama más genérico.

2 Tal limitación tiene que ver con la necesidad de acotar un volumen verdaderamente amplio de publicaciones: la base de datos manejada fue de 387 obras pertenecientes a 115 narradores/as. La fecha inicial (1975) coincide con un momento de elevada importancia política y cultural; las publicaciones posteriores al año 2000 se incluyen en nota.

${ }^{3}$ No hablaremos de los autores más jóvenes, de las nuevas generaciones que publican su obra a partir de los años noventa (vid. mi tesis de doctorado previamente citada): Antonio Muñoz Molina, Almudena Grandes, Lucía Etxebarría o Alicia Giménez Bartlett, por citar sólo algunos de ellos. Diremos sólo que sus numerosas publicaciones ofrecen optimistas perspectivas a la publicación de nuestra narrativa en Italia. 
puestas plantea al escritor una realidad no siempre fácil de afrontar ${ }^{4}$; pero a pesar de la pluralidad de tendencias, hay un tronco original: la voluntad de contar historias, no importa de qué tipo. Nos centraremos, por tanto, en los narradores más significativos ${ }^{5}$ que publican su obra en Italia, aquéllos que muchos encuadran en corrientes posmodernistas, para tocar después, a través de figuras ciertamente representativas, dos géneros concretos: el policiaco y el de aventuras; no incluiremos en el presente artículo lo que no constituye en sí una tendencia narrativa, sino una polémica agrupación, la narrativa femenina, que ha sido ya objeto de otro trabajo (N. Pérez Vicente, 2005)

Hay varios autores que, a pesar de ser muy conocidos y editados en España, están presentes en el panorama italiano a través de una sola publicación. Es el caso de Luis Mateo Díez con I mali minori [Los males menores] (L. M. Díez, 1996), conjunto de microrrelatos que no coincide exactamente con la obra original del mismo título (1993), ya que no incluye la colección Álbum de esquinas, constituida por siete cuentos de mayor extensión, que abría el volumen. Éste responde, creemos, al explícito deseo de la pequeña editorial Il Melangolo que, dirigida al mundo universitario (surge por iniciativa de la Universidad de Génova), se plantea un criterio de fuerte compromiso cultural: de hecho la autora del epílogo y traductora, Maria Vittoria Calvi, es profesora universitaria en Milán, a la vez que se trata de uno de los pocos volúmenes pertenecientes a esta etapa que cuentan con aparato crítico. No parece que el hecho de que el autor se integre en la llamada «escuela leonesa» - a la que pertenece también Julio Llamazares, como veremos posteriormente - haya contribuido a una mayor difusión, aunque el regionalismo y la especial atención a los grupos de cultura (e incluso de lengua) autóctona es un factor usado muchas veces para atraer al lector. Por otra par-

\footnotetext{
4 «Al benessere economico, alla recuperata libertà democratica, alla pluralità culturale non si accompagna una parallela soddisfazione interiore. Anzi, nei tanti 'mondi im-possibili' di questa letteratura, il paese d'origine, la comunità sociale, il nucleo familiare si svuotano di relazioni significative, diventano terreno di nessuno, in cui personaggi inetti e disorientati si dimostrano più inclini a subire che a fare, a patire che a agire. Il destino soggettivo tramonta, il senso oggettivo si oscura [...]. Dubbio, instabilità, ambivalenza sono dunque gli effetti di un'accentuata disgregazione dell'identità e di un forte sentimento di estraniazione che affievoliscono i rapporti costruttivi con il mondo» (E. Pittarello, 1995: 60).

5 A pesar de los numerosos autores, no hay que olvidar que otros muchos están ausentes (Juan Pedro Aparicio, José Antonio Gabriel y Galán, José María Guelbenzu, Manuel Longares, Jorge Martínez Reverte, Marina Mayoral, Vicente Molina Foix, Lourdes Ortiz, Raúl Guerra Garrido, Soledad Puértolas, Fernando G. Delgado, Terenci Moix, y un largo etcétera) o aparecen sólo formando parte de alguna antología.

${ }^{6}$ En él se habla concretamente de Cristina Fernández Cubas, Adelaida García Morales, Ana María Moix, Rosa Montero, Rosa Regás, Carmen Rico-Godoy, Maruja Torres y Esther Tusquets. Su presencia editorial no es muy fuerte: todas ellas publican como mucho dos volúmenes.
} 
te, este conjunto de textos breves es un buen ejemplo de literatura «en píldoras», potencialmente más vendible.

Antonio Gala, de igual modo, es conocido sólo a través La passione turca [La pasión turca] (1993) (A. Gala, 1995), mientras que queda inédita su producción dramática ${ }^{7}$ y poética. De todos los best-seller posteriores a 1990 se publica el de mayor carga erótica (apoyada por la imagen de portada, y la contraportada, que reproduce el texto de uno de los fragmentos más «ardientes» de la novela), situado en un ambiente exótico y en torno a un personaje femenino. Todo ello es garantía de un éxito comercial (de hecho es un volumen sin aparato crítico ni notas) que la potente editorial Rizzoli no deja de aprovechar. Es extraño, sin embargo, que no se hayan publicado más novelas del autor, en concreto las de tinte histórico. No hay que olvidar, por otra parte, que esta obra ha sido llevada al cine, y el factor «arrastre» (J. Peñate Rivero, 1997) es siempre importante para la publicación de un libro.

La única traducción de nuestro premio Cervantes 2002, José Jiménez Lozano, es de tinte diametralmente opuesto: Sara di Ur [Sara de Ur] (J. Jiménez Lozano, 1993) es una historia de inspiración bíblica que ve la luz en Italia debido al interés específico del editor, Danilo Manera, y al criterio de la editorial, Biblioteca del Vascello, que privilegia la publicación de obras pertenecientes a las literaturas menos conocidas en Italia. Cuenta con la ayuda del Ministerio de Cultura español, y es un volumen cuidado, anotado y con apoyo crítico. El criterio de traducción utilizado, el semántico (P. Newmark, $\left.1995^{8}\right)$, confirma todo lo anteriormente dicho. De mayor difusión es el volumen de Luis Landero, su fundamental Juegos de la edad tardía (1989), Giochi tardivi (L. Landero, 1991), premio de la Crítica y Nacional de Literatura 1991, publicado en Feltrinelli. Scorpioni Coggiola (1995: 284) opina que el ser «piuttosto macchinoso e prolisso» no le ha impedido un «grande successo di pubblico e di critica». También Juan José Millás, considerado por muchos el mejor representante de las ansias del individuo posmoderno y definido por Elide Pittarello (1995: 54) como «uno degli autori che più stanno contribuendo a mostrare il lato sommerso del mondo», está presente con una de sus novelas de más éxito: El desorden de tu nombre (1988) [Il disordine

\footnotetext{
7 A excepción de Anelli per una dama (Roma: Empiria, 1994; tr. Sara Zanghi).

${ }^{8}$ A partir de ahora utilizaremos la conocida nomenclatura de Peter Newmark (1995), que diferencia dos principales criterios de traducción: el semántico (que hace hincapié en la lengua del texto original) y el comunicativo (que lo hace en la lengua de llegada). Por falta de espacio, para más aclaraciones remitimos al autor.
} 
del tuo nome] (J. J. Millás, 1994) ${ }^{9}$. Sin embargo, la existencia de esta edición es, creemos, más bien casual: de hecho se publica en Cronopio, joven editorial que se centra en temas de filosofía, ética, literatura en general, política y ensayo y se dirige a un público no especializado. Responde muy probablemente a la decisión personal de los editores, o a la propuesta de Maria Rosaria Alfani (Universidad de Nápoles), interesada en un autor representativo de los ochenta que se caracteriza por un especial uso de la lengua.

La única publicación de Manuel Vicent, escritor, crítico de arte y periodista, es un libro de viajes, de 1994, de título bastante largo (Del Café Gijón a Ítaca. Descubrimiento del Mediterráneo como mar interior), abreviado en la edición italiana: Mediterraneo, mare interiore (M. Vicent, 1995). No se conocen en cambio importantes novelas como La balada de Caín (premio Nadal 1986) o Tranvía a la Malvarrosa (1994), llevada al cine por José Luis García Sánchez en 1996. Vicent comparte colección (Traveller Feltrinelli) con otros autores españoles: Maruja Torres y Julio Llamazare ${ }^{10}$. En este caso, como sostiene la reseña de Guido Bonino (1995: 34), se trata de un viaje espacial, cultural e interior, que podría incluirse también entre los numerosos libros sobre la cultura, historia y tradiciones del Mediterráneo. Todo ello hace pensar que lo que condiciona su publicación no es el autor, desconocido en Italia, sino su género: el auge de los libros de viajes.

Hay otros escritores, generacionalmente anteriores, que, sin embargo, suelen ser incluidos en la «nueva narrativa», ya que participan del mismo espíritu y se dan a conocer principalmente a partir de los años ochenta. Nos referimos en primer lugar, a José Luis Sampedro, economista de profesión que no alcanza el éxito hasta 1981 con Octubre, Octubre (inédita en Italia). Il sorriso etrusco [La sonrisa etrusca] (1985) (J. L. Sampedro, 1997, 1999), que en España tuvo hasta 1992 un total de 41 ediciones $^{11}$, aparece en dos editoriales diferentes. Situada en Milán, la obra se centra en la relación de un anciano ex-partisano con su nieto, lo cual pudiera explicar el interés causado en Italia. Il Saggiatore, que ya había publicado una obra de economía de Sampedro $^{12}$, decide lanzarse al descubrimiento de otras facetas del escritor,

9 Posteriormente han aparecido L'ordine alfabetico (Milano: Il Saggiatore, 2001; tr. Paola Tomasinelli); Non guardare sotto il letto (Milano: Il Saggiatore, 2002; tr. Paola Tomasinelli) y Racconti di adulteri disorientati (Torino: Einaudi, 2004; tr. Paola Tomasinelli).

${ }^{10}$ Maruja Torres, Amor America. Un viaggio sentimentale in America Latina (Milano: Feltrinelli, 1994; tr. Pino Cacucci e Gloria Corica) y Julio Llamazares, Tras-os-montes: un viaggio portoghese (Milano: Feltrinelli, 1999; tr. Elena Liverani).

11 Más adelante se publica La vecchia sirena (Milano: Il Saggiatore, 2001; tr. Roberta Bovaia).

${ }^{12}$ Le forze decisive dell' economia mondiale (Milano: Il Saggiatore, 1967; tr. Clara Mainoldi). 
aunque no es una editorial especialmente centrada en narrativa. Tropea, perteneciente al grupo «Il Saggiatore», aprovecha la misma traducción para publicarlo en EST, una colección de libros de bolsillo. Son volúmenes divulgativos, sin aparato crítico ni notas.

En el mismo caso se encuentra Juan Eduardo Zúñiga. Sólo hay dos libros de relatos de este escritor madrileño, especialista en literaturas eslavas: $L ' a$ nello di Puškin [El anillo de Pushkin] (1983) (J. E. Zúñiga, 1994b) y La terra sarà un paradiso [La tierra será un paraíso] (1989) (J. E. Zúñiga, 1994a). Como en otras ocasiones, el mérito de su descubrimiento en Italia es del editor, Danilo Manera, y de Biblioteca del Vascello que, dedicada a sacar a la luz a los autores menos conocidos, decide en este caso descubrir a un autor que con su postura testimonial y crítica, más bien pesimista, revisa diversos aspectos de la historia española, concretamente la guerra civil y la posguerra. Recordemos además que Zúñiga cuenta con pocas publicaciones en España, así que el hecho de que en Italia se editen dos volúmenes suyos no es un mal porcentaje. Sus traductores, por otra parte, siguen preferentemente una orientación semántica, lo cual es acorde con este tipo de publicación minoritaria que cuenta además con aparato crítico. Es también significativo que una de sus obras, L'anello di Puškin, sea objeto de una traducción que podríamos llamar «coral», realizada por un grupo universitario que considera ésta última un proceso de colaboración y experimentación común.

Otro de los autores presentes a través de dos títulos es Félix de Azúa, caracterizado por la crítica a través de líneas fácilmente identificables como «posmodernistas», del cual se publica: Storia di un idiota narrata da lui stesso [Historia de un idiota contada por él mismo o el contenido de la felicidad] (1986) (F. de Azúa, 1990) - llevada con éxito a los escenarios en adaptación teatral- y Cambio di bandiera (F. de Azúa, 1993) [Cambio de bandera] (1991), situada en la guerra civil ${ }^{13}$. Tal como ocurre con otros escritores (por ejemplo, con Juan Marsé o Juan Goytisolo en los años sesenta ${ }^{14}$ ), se evitan las obras de su primera etapa, textos autorreflexivos de enorme complejidad (Las lecciones de Jena, 1972 o Las lecciones suspendidas, 1974), para pasar directamente a su línea posterior, mucho más irónica. No se conoce en cambio su labor poética, a excepción de su participación en la famosa antología de Castellet ${ }^{15}$. Ambas obras se traducen en editoriales fuertes (Garzanti y Guan-

13 Se traduce también un cuento infantil, Il bambino che parlava agli animali (Milano: Mondatori, 1997; tr. Francesca Lazzarato).

14 Ver mi tesis de doctorado citada (nota 1).

15 Nueve novísimos poetas españoles (1970) —edición italiana: Giovani poeti spagnoli (Milano: Einaudi, 1975; tr. Rosa Rossi)—. 
da) mediante un criterio semántico. En su introducción en Italia ha influido, sin duda, el éxito teatral obtenido en Francia por la versión de una de sus novelas, con un éxito paralelo al de otros tres escritores barceloneses que también estrenan en dicho país: Javier Tomeo, Manuel Vázquez Montalbán y Eduardo Mendoza. Danilo Manera (1991), sin embargo, se pregunta el porqué del impacto en España de este desengañado retrato generacional, explicable quizá por el sarcasmo y los continuos «guiños» culturales que en buena medida se le escapan al lector extranjero: «è difficile evitare l'impressione di imbarazzo quando l'autore calca la mano su luoghi comuni in veste filosofica, militante professione d'inettitudine, autoironia e altri esorcismi privati», lo cual le lleva a concluir que «resta più interessante come saggista» (D. Manera, 1991: 30).

Scorpioni Coggiola (1995: 327) considera a Álvaro Pombo importante exponente del «romanzo psicologico» que «usa l'invenzione narrativa come mezzo per esplorare l'antinomia realtà/ apparenza o, meglio, per evidenziare la confusione tra ciò che si immagina e ciò che è». Y ello, sobre todo, gracias a una novela, El héroe de las mansardas de Mansard (1983; Premio Herralde), que narra las experiencias de un niño que vive en un mundo de invenciones: L'eroe delle mansarde di Mansard (A. Pombo, 1987). Hay una segunda traducción, Dove le donne (A. Pombo, 2000) [Donde las mujeres] (1996), muy posterior, lo cual hace pensar que no hay línea común que la una a la primera. Aparecen en dos editoriales de grandes dimensiones (Einaudi y Garzanti) que conceden mucho espacio a la literatura contemporánea y tienen fines más bien divulgativos, de hecho ambas carecen de aparato crítico y sólo una está brevemente anotada. Contamos igualmente con dos títulos del «eccentrico, elegante e personalissimo scrittore catalano» (B. Arpaia, 2002: 37). Enrique Vila-Matas, cuya prosa irónica y llena de referencias metaliterarias ha sido definida por muchos como «posmoderna». La primera es una novela de reducidas dimensiones que obtuvo un gran éxito: Storia abbreviata della letteratura portatile [Historia abreviada de la literatura portátil] (1985) (E. Vila-Matas, 1989); la segunda es un conjunto de relatos titulado Suicidi esemplari [Suicidios ejemplares] (1991) (E. Vila-Matas, 1994). Sellerio, editorial que siempre ha escogido mucho a sus autores, apuesta por este escritor no siempre fácil de entender, aunque la ausencia de aparato crítico y de notas sugiere la orientación hacia un público amplio ${ }^{16}$.

${ }^{16}$ Constatamos la existencia de otras publicaciones más recientes que confirman un creciente interés por el narrador: Bartleby e compagnia (Milano: Feltrinelli, 2002; tr. Danilo Manera); La assassina letterata (Roma: Voland, 2004; tr. Danilo Manera y Elisabetta Pagani); Suicidi esemplari (Roma: Nottetempo, 2004; tr. L. Panunzio Cipriani; revisione, Fiammetta Biancatelli) y Il mal di Montano (Milano: Feltrine1li, 2005; tr. Natalia Cancellieri). 
Pasemos a continuación a los autores del periodo más publicados en Italia. Casi todas las ediciones de Javier Marías destacan que enseñó en Oxford y en EEUU; que además de escritor de novelas, ensayos y cuentos es traductor; que ha recibido infinidad de premios y que ha obtenido un aplastante éxito en más de treinta países, habiendo sido traducido a más de veinticinco idiomas. Se destaca, por otra parte, la calidad de su prosa y el análisis introspectivo de los personajes, así como el valor de la incertidumbre que se esconde tras la aparente intriga, aspecto en cambio meramente circunstancial; del mismo modo se señalan los rasgos metaliterarios de su escritura. Pero su éxito le precede, y ello favorece que haya un total de cinco novelas del autor aparecidas en diversas editoriales, con diversas reediciones ${ }^{17}$. Se trata de L'uomo sentimentale [El hombre sentimental] (1986) (J. Marías, 2000a); Tutte le anime [Todas las almas] (1989) (J. Marías, 1999b; 1999c); Un cuore così bianco [Corazón tan blanco] (1992) (J. Marías, 1996; 1999a) con dos traductores diferentes, en un lapso de tiempo muy breve; Domani nella battaglia pensa a me [Mañana en la batalla piensa en mí] (1994) (J. Marías, 1998a; 1998b) y Nera schiena del tempo [Negra espalda del tiempo] (1998) (J. Marías, 2000b). Aparte de Donzelli, pequeña editorial de cultura que pretendía llenar el vacío cultural de los años ochenta, las demás publicaciones están en la poderosa Einaudi, una de las mayores firmas italianas, y pocas tienen aparato crítico: Marías parece considerarse un «producto» que vende por sí solo. Es interesante observar que su penetración en Italia comienza con las obras contemporáneas en el momento de la publicación - Corazón tan blanco - y sigue, «hacia atrás» con otras más antiguas que se rescatan ante la seguridad de una respuesta afirmativa del público. Así, se publican Todas las almas —ésta, con críticas de todo tipo, incluso algunas que lo tachan de «machismo» (M. Nicola, 1999), viene apoyada por la polémica producción cinematográfica de los Querejeta, titulada El último viaje de Robert Rylands (1996), que tantas disputas valió a estos últimos con el autor-y El hombre sentimental, de 1989 y 1986 respectivamente.

Eduardo Mendoza cuenta con seis publicaciones, además de su drama Restauració (1990), traducido al castellano en $1991^{18}$. Entre ellas se en-

17 Tras el 2000, además de numerosas reediciones, siguen apareciendo sus recientes éxitos, entre ellos también ensayos: Quand' ero mortale (Torino: Einaudi, 2001; tr. Glauco Felici); Malanimo (Torino: Einaudi, 2001; tr. Paola Tomasinel1i); Vite scritte (Torino: Einaudi, 2004; tr. Glauco Felici); Il tuo volto domani. Vol. I: Febbre e lancia (Torino: Einaudi, 2003; tr. Glauco Felici); Selvaggi e sentimentali. Storie di calcio (Torino: Einaudi, 2002; tr. Glauco Felici) y Attraversare l'orizzonte (Torino: Einaudi, 2005; tr. Glauco Felici).

${ }^{18}$ Restaurazione [Restauració] (Messina: A. Lippolis, 1996; tr. e intr. Cecilia Galzio), cuya representación en lengua francesa tuvo un gran éxito en 1990. 
cuentra aquella que abriría nuevos horizontes a la forma de narrar en España, La verdad sobre el caso Savolta (1975; premio de la Crítica) (E. Mendoza, 1995), que, según Scorpioni Coggiola (1995: 158), «[...] segna nel 1975 l'inizio di una nuova fase dell'evoluzione narrativa [...]. Non crea una corrente letteraria determinata; rappresenta, piuttosto, la prima vera reazione al vuoto argomentale del romanzo; costituisce, inoltre, il primo esempio degno di nota sia dell'estetica postmoderna, sia della finzione testimoniale indiretta nei riguardi dei problemi sociali». Hay otras publicaciones de tipo policiaco, como Il mistero della cripta stregata [El misterio de la cripta embrujada] (1979) (E. Mendoza, 1990); La città dei prodigi [La ciudad de los prodigios] (1986) (E. Mendoza, 1987), novela de grandes dimensiones en la que la ciudad de Barcelona es la protagonista indiscutible; L' isola inaudita [La isla inaudita] (1989) (E. Mendoza, 1991), cuya trama se sitúa en una Venecia donde se mezclan los sueños y la realidad; Nessuna notizia di Gurb [Sin noticias de Gurb] (1991; aparecido en 1990 por entregas en El País) (E. Mendoza, 1992), con ingredientes de humor; y L'anno del diluvio [El año del diluvio] (1993) (E. Mendoza, 1994), provocativa historia de amor situada en la posguerra que aparece en la genovesa Le Mani, dirigida a un público culto no especialista. Como vemos, es un panorama muy variado del que el mundo editorial italiano se hace eco ${ }^{19}$. Igual que otros autores, Mendoza es introducido en el panorama editorial italiano «con pies de plomo»: la primera en atreverse es Longanesi, firma medio-grande, de mucha fuerza, que presenta la recién premiada (entre otros con el prestigioso «Grinzane Cavour») La ciudad de los prodigios. Quien toma el relevo es Feltrinelli, que se arriesga, en primer lugar, con una obra de años atrás (El misterio de la cripta embrujada), de temática policiaca y mucha influencia de la novela americana de género, ya conocida por el lector italiano; y en segundo lugar, con otras más cercanas. Sólo entonces, con el terreno ya muy fertilizado, se lanza con una novela anterior a las ya publicadas, La verdad sobre el caso Savolta, que, a pesar de todo el interés crítico despertado, no se presenta en Italia como caso literario, sino exclusivamente como novela policiaca con elementos de folletín y, por si acaso, se «disfraza» a través de una portada que muestra un desnudo femenino: quizá los editores tenían serias dudas sobre la posible venta del libro.

Aunque es algo mayor que otros narradores citados y empezó a publicar en los sesenta, el reconocimiento le llega a Javier Tomeo (como a Sampedro

${ }_{19}$ Sin embargo, aparte de alguna reedición, hay una sola publicación nueva: Il tempio delle signore (Milano: Feltrinelli, 2002; tr. M. Finassi Parolo). 
y a Zúñiga) a partir de los ochenta. Hay un número considerable de obras suyas publicadas en Italia, «testi che hanno contribuito al rinnovamento del romanzo spagnolo nelle ultime decadi» (L. B. Porto Bucciarelli, 1990: 226): I nemici [Los enemigos] (1974) (J. Tomeo, 1992), que pasó bastante desapercibida en su época, aunque luego fue reeditada; Il castello della lettera cifrata [El castillo de la carta cifrada] (1979) (J. Tomeo, 1991), llevada al teatro en 1997, que tuvo un gran éxito de crítica y le abrió las puertas de las letras españolas; Il maggiordomo miope [El mayordomo miope] (1989) (J. Tomeo, 1993) y Amato mostro [Amado monstruo] (1985) (J. Tomeo, 1989), también adaptadas al teatro; y las más recientes, Dialogo in re maggiore [Diálogo en re mayor] (1980) (J. Tomeo, 2000) y Il delitto del cinema Oriente [El crimen del cine Oriente] (1995) (J. Tomeo, 1999) —ésta última con una adaptación cinematográfica ese mismo año-. Se publica también un volumen de cuentos, Problemi oculari [Problemas oculares] (1990) (J. Tomeo, 1998), divertida y a la vez dramática obra en la que una sarta de personajes miopes funcionan como modelo de la existencia humana.

Siete títulos que se distribuyen en editoriales bastante diversas entre sí y que no se reeditan. Comienza Mondadori con Amado monstruo (1989), una de sus novelas más difundidas y de mayor impacto; Porto Bucciarelli (1990: 227) la describe así: «un romanzo inconsueto, dall'atmosfera sconcertante e surreale che ventila in una situazione speculare i difficili rapporti tra madri possessive e figli sottomessi». Las siguientes aparecen en Bollati Bollinghieri, editorial de tipo medio que recuperará obras anteriores. En cambio, son dos firmas de pequeña dimensión las que se ocupan de las novelas más modernas: Il Melangolo, de la universidad de Génova, que ya publicara los relatos breves de Luis Mateo Díez; y Passigli, de muy buena difusión. Son volúmenes con poco aparato crítico y sin notas, no dirigidos a un público especializado.

Varios factores influyen en la penetración de Tomeo en el panorama editorial italiano: una temática cercana al thriller, su uso del humor y la ironía para retratar al género humano (y en el caso de Amado monstruo el tema concreto de la mujer - la madre - como castradora del hombre), además de las dimensiones breves tanto de sus novelas como de sus libros de relatos - aunque se trate de una brevedad sólo superficial (R. Acín, 1992), resultado de una condensación conceptual—. No hay que desestimar tampoco como ingrediente de éxito su relación con Kafka e incluso con el Buñuel surrealista. También es evidente que su fama fuera de España, sus numerosas versiones teatrales y cinematográficas, apoyan sin duda la presencia de su obra en Italia. Es más: el tardío descubrimiento en España tiene sin duda que 
ver con su éxito en el extranjero ${ }^{20}$. Por otro lado, alejado tanto del realismo de posguerra como del experimentalismo, sus novelas cortas - poco estimadas en un momento en que se prefería mayor extensión- comienzan a conocerse sólo al afianzarse en los ochenta una renovación narrativa que lo acerca a los escritores más jóvenes que él.

Aunque hay varios autores policiacos traducidos al italiano en el periodo estudiado $^{21}$, vamos a centrarnos en uno de ellos, Manuel Vázquez Montalbán. Su caso es verdaderamente especial, ya que es, sin duda, uno de los españoles del siglo XX más publicados en Italia: entre ensayo ${ }^{22}$, material ecléctico como su primerizo Manifiesto subnormal (1970) - Manifesto subnormale (M. Vázquez Montalbán, 1980)—, libros de recetas que aprovechan el éxito del personaje Carvalho, poesía ${ }^{23}$ y, sobre todo, novela, contamos con unas cincuenta obras que ven la luz en este país entre 1975 y 2000, lo cual supone el grueso de toda la producción literaria del autor. Este éxito parece tener mucho que ver con el género en el que se suele encuadrar la mayoría de sus obras, el giallo o policiaco, y con la orientación crítica y testimonial de la sociedad moderna que Vázquez Montalbán les da. Porque, «come ben sanno y lettori di Montalbán, la trama poliziesca è solo una componente dei suoi romanzi, dei quali si è detto che riflettono 'le questioni nodali della Spagna postfranchista'» (C. Biondi, 1996: 18) ${ }^{24}$.

La narrativa publicada en Italia es la siguiente: en cuanto a novelas, $\mathrm{Ta}$ tuaggio [Tatuaje] (1975) (M. Vázquez Montalbán, 1991c); La solitudine del manager [La soledad del manager] (1978) (M. Vázquez Montalbán, 1993c); Un delitto per Pepe Carvalho [Los mares del sur] (1979) (M. Vázquez Montalbán, 1982) — publicado más adelante con el título I mari del sud (M. Váz-

\footnotetext{
20 Según Acín (1992), el éxito alemán de El castillo de la carta cifrada y la repercusión francesa de Amado monstruo es lo que produce que se le empiece a considerar en España.

${ }^{21}$ Juan Luis Cebrián, Francisco González Ledesma, Juan Madrid y José María Riera de Leyva. Vid. mi tesis de doctorado citada (nota 1 ).

22 Hay muchos publicados entre 1975 y 2000: L'informazione (Novara: Istituto Geografico di Agostini, 1977; tr. Alvise Cerati); Pasionaria e i sette nani (Milano: Frassinelli, 1997; tr. Hado Lyria); E Dio entró all' Avana (Frassinelli, 1999; tr. Hado Lyria; «Saggistica»); Lo scriba seduto (Milano: Frassinelli, 1997; tr. H. Lyria); Paul Gauguin: la lunga fuga (Firenze: Passigli, 1998; tr. Hado Lyria); Pamphlet dal pianeta delle scimmie (Milano: Feltrinelli, 1995); Marcos: il signore degli specchi (Milano: Frassinelli, 2001; Milano: Mondolibri, 2001); Storie di padri e figli (Milano: Feltrinelli, 2001) y Questioni marxiste (Milano: Frassinelli, 2001).

${ }^{23}$ Città / Ciudad [Ciudad] (Milano: Frassinelli, 1997; Milano: Mondadori, 1998; ambas ed. Hado Lyria). Anteriormente José María Castellet lo había incluido en su importante antología (Vid nota 14).

${ }^{24}$ Se puede hablar de un auténtico fenómeno literario que ha dado lugar incluso a volúmenes de recopilación, como Le Barcellone di Pepe Carvalho, de C. Alberto Giorgio Cassani, incluido en la colección «Città letterarie» (Milano: Unicopli, 2000), y el ensayo de Quim Aranda, Piacere, Pepe Carvalho. Biografia autorizzata dell' investigatore più famoso di Spagna (Milano: Feltrinelli, 1997; tr. Silvia Meucci).
} 
quez Montalbán, 1994b)_; Assassinio al Comitato Centrale [Asesinato en el Comité Central] (1981) (M. Vázquez Montalbán, 1984); Gli uccelli di Bangkok [Los pájaros de Bangkok] (1983) (M. Vázquez Montalbán, 1990b); La rosa di Alessandria [La rosa de Alejandría] (1984) (M. Vázquez Montalbán, 1995a, 1996c); Il pianista [El pianista] (1985) (M. Vázquez Montalbán, 1990c); Le terme [El balneario] (1986) (M. Vázquez Montalbán, 1996b); Quartetto [Cuarteto] (1987) (M. Vázquez Montalbán, 1990a, 1996a); Gli allegri ragazzi di Atzavara [Los alegres muchachos de Atzavara] (1987) (M. Vázquez Montalbán, 1993a); Il centravanti è stato assassinato verso sera [El delantero centro fue asesinado al atardecer] (1988) (M. Vázquez Montalbán, 1991a); Galíndez [Galíndez] (1990) (M. Vázquez Montalbán, 1991b); Il labirinto greco [El laberinto griego] (1991) (M. Vázquez Montalbán, 1992b); Io, Franco [Autobiografía del General Franco] (1992) (M. Vázquez Montalbán, 1993b); Lo strangolatore [El estrangulador] (1994) (M. Vázquez Montalbán, 1995b, 1995c); Il premio [El premio] (1996) (M. Vázquez Montalbán, 1998b, 1999c); Quintetto di Buenos Aires [Quinteto di Buenos Aires] (1997) (M. Vázquez Montalbán, 1999a); O Cesare o nulla [O César o nada] (1998) (M. Vázquez Montalbán, 1998a); Riflessioni di Robinson davanti a centoventi baccalà [Reflexiones de Robinsón ante un bacalao] (1998) (M. Vázquez Montalbán, 2000a) y L’uomo della mia vita [El hombre de mi vida] (2000b) (M. Vázquez Montalbán, 2000). Podemos considerar narrativa breve sus particulares libros de recetas: Ricette immorali [Recetas inmorales] (1988) (M. Vázquez Montalbán, 1992a) y Le ricette di Pepe Carvalho [Las recetas de Pepe Carvalho] (1989) (M. Vázquez Montalbán, 1994c); además de tres volúmenes de cuentos: Dallo spillo all' elefante [Pigmalión y otros relatos] (1987) (M. Vázquez Montalbán, 1994a), Storie di fantasmi [Historias de fantasmas] (1987) (M. Vázquez Montalbán, 1999b) y Il fratellino [El hermano pequeño] (1994) (M. Vázquez Montalbán, 1997) 25.

El éxito de este autor ha ido in crescendo a lo largo del periodo estudiado. La primera obra traducida (aparte de Manifiesto subnormal, un texto casi de

${ }^{25}$ Hay un volumen de narrativa juvenil: Il signore dei bonsai (Milano: Feltrinelli, 2000; tr. Hado Lyria; il. Lluïsa Jover). Sus publicaciones posteriores al 2000, ensayos incluidos (sin nombrar las numerosas reediciones), son numerosas, lo cual hace pensar que su éxito continúa tras su muerte: Ho ammazzato a J. F. Kennedy (Milano: Feltrinelli, 2001; Tr. Hado Lyria); Questioni marxiste (Milano: Frassinelli, 2001); Erec e Enide (Milano: Frassinelli, 2002; tr. H. Lyria); Happy end. Ma la storia non finisce qui (Milano: Frassinelli, 2003; Milano: Mondolibri, 2004); Tre storie d' amore (Milano: Feltrinelli, 2003; Hado Lyria); Il centravanti è stato assassinato verso sera (Roma: Gruppo editoriale L'Espresso, 2003) (Roma: La Repubblica, 2004; tr. L. Panunzio Cipriani); Milenio. Pepe Carvalho sulla via di Kabul. Vol. I (Milano: Feltrinelli, 2004; tr. Hado Lyria); Il potere e la boria (Milano: Frassinelli, 2004; tr. Hado Lyria) y Assassinio al Comitato Centrale (Milano: Feltrinelli, 2005; tr. L. Panunzio Cipriani). 
culto) es Los mares del Sur. El descubridor es Editori Riuniti, de fuerte componente político al haber sido durante años la editorial oficial del Partido Comunista. La traducción, que utiliza un criterio comunicativo moderado (aunque cambia significativamente el título de la novela, dirigido a dar a conocer un «producto» que todavía no se había introducido en el mercado editorial italiano), se encarga a Sonia Piloto de Castri, que no volverá a traducir a Montalbán. La obra se reeditará doce años después en Feltrinelli, cuando su éxito ya es evidente, esta vez con su título original, en traducción de Hado Lyria. Pasan dos años hasta que Sellerio (que sólo presentará al autor una vez más, con El pianista), en 1984, se lanza con Asesinato en el Comité Central: Panunzio Cipriani, con un criterio comunicativo, tampoco traducirá de nuevo al escritor. El tercer invitado a la escena editorial es Feltrinelli, con Los pájaros de Bangkok (1990). Ésta es la firma que ostentará, alternándose con Frassinelli, el monopolio de las ediciones en Italia, estabilizando la situación y homogeneizando la receptividad al emplear ambas siempre a la misma traductora, Hado Lyria ${ }^{26}$. Pero Feltrinelli no se valdrá todavía para este primer volumen de ella, sino de Sandro Ossola que, con un criterio comunicativo, tampoco repite la experiencia. Hasta aquí transcurren pocos años entre el original y la publicación italiana. Son sus grandes éxitos, reconocidos internacionalmente con numerosos premios, los que se publican casi inmediatamente, como Galíndez (1990) con el que hará su aparición Frassinelli, en 1991. A partir de entonces las ediciones retroceden para rescatar obras ya publicadas en España, con el fin de que participen del habitual éxito del autor. Es el caso de El pianista (1985), Los alegres muchachos de Atzavara (1987), etc.

A partir de 1998, año en que se cumple el veinticinco aniversario de la primera aventura de Carvalho, observamos una diferencia: Vázquez Montalbán se ha situado en los primeros puestos de las listas de ventas en varias ocasiones y ya se han publicado sus mejores novelas: se empieza, por tanto, a seguir día a día los acontecimientos editoriales. De hecho $O$ César o nada, El premio, Quinteto de Buenos Aires, etc., se publican en Italia casi inmediatamente después de su debut en España. Es más, comienzan a proponerse obras de recopilación que aprovechan el éxito del autor, como los libros de recetas y los de cuentos. Paralelamente, no hay que olvidarlo, siguen publicándose sus ensayos.

Ni que decir tiene que este boom editorial coincide con un interés también enorme por parte de la crítica: es uno de los autores españoles más reseñado

${ }^{26}$ Y dividiéndose de algún modo las publicaciones: la serie Carvalho, Feltrinelli; el resto de la narrativa, Frassinelli. 
y es también difícil encontrar a otro que tenga más ediciones y reediciones. En ese sentido Vázquez Montalbán se asimila a los más vendidos en Italia, a los creadores de best-seller del momento, de los cuales, recordémoslo, es el único español. Ni siquiera García Lorca iguala su fama, aunque uno y otro respondan a fenómenos muy diferentes. Lo que está claro es que en Italia parece considerársele el paradigma del narrador actual. Además, hay continuas referencias al autor en los volúmenes más variados (de Almudena Grandes, González Ledesma, etc.), señal de la fuerza del «factor arrastre» (J. Peñate Rivero, 1997) que Montalbán ha producido. Recapitulando: este «Marlowe» español gusta porque no es sólo un giallo, sino que la referencia a los problemas sociales y culturales de una nueva España pueden llegar a ser identificados por el lector con los de toda Europa. El tiempo nos dirá si su prematura muerte podría cambiar las tornas de la situación, aunque no parece que ello haya sucedido de momento ${ }^{27}$.

Nos referiremos por último al género de aventuras, centrándonos en un único autor ${ }^{28}$ : Alberto Vázquez Figueroa. Pocos volúmenes de historia de la literatura española lo incluyen, pero es uno de los considerados «fabricantes» de best-seller que, aunque no aparecen nunca en listas de ventas, tiene, como afirma Jordi Gracia (2000: 30), un público masivo y fiel. De sus más de cuarenta novelas publicadas, nueve lo están en Italia, cada una en diversas editoriales y con reediciones. Las obras son Ashanti. La via degli schiavi [Ébano] (1975) (A. Vázquez Figueroa, 1978, 1979a, 1991); Manaos [Manaos] (1975) (A. Vázquez Figueroa, 1979b, 1980b, 1992); Come un cane rabioso [Como un perro rabioso] (1976) (A. Vázquez Figueroa, 1981); Panama Panama [¡Panamá, Panamá!] (1977) (A. Vázquez Figueroa, 1979c, 1980a); Tuareg [Tuareg] (1981) (A. Vázquez Figueroa, 1983, 1994); Il re delle iguane [La iguana] (1982) (A. Vázquez Figueroa, 1985a, 1985b); La ragazza del mare [Océano] (1984) (A. Vázquez Figueroa, 1986a, 1986b); Il fiume nero dei diamanti [Maradentro] (1985) (A. Vázquez Figueroa, 1999) y Yàiza [Yaiza] (1984) (A. Vázquez Figueroa, 1998). La editorial que se ocupa casi mayoritariamente del autor es Sperling \& Kupfer, basada desde su fundación en obras extranjeras. Es significativo que muchas de las novelas vuelven a editarse en Sperling \& Paperback que, perteneciente al mismo grupo Sperling \& Kupfer-Frassinelli, se ocupa sólo de ediciones en paperback. Operación semejante es la realizada por «Club degli Editori», que, como sabemos, vuelve a editar los mayores

27 Vid. nota 24.

${ }^{28}$ Otros que publican en el mismo periodo novelas históricas y de aventuras son Vicente Escrivá, Juan Eslava Galán, Fernando Sánchez Dragó y Juan Antonio Vallejo-Nágera. Vid. tesis citada (nota 1). 
éxitos de ventas. El traductor «oficial» parece ser Stefano Bossi, y un misterioso «M. A.» se ocupa de las tres últimas editadas. El hecho de que no se nos dé el nombre completo del traductor, unido a que en todos los volúmenes consultados éste aparece siempre camuflado entre los créditos, nos demuestra la poca importancia que se da en tal tipo de ediciones, muy divulgativas, a su figura ${ }^{29}$. Nótese, por otra parte, cómo los títulos italianos cambian notablemente respecto al original español. Es decir, el concepto de «texto» queda en estas ediciones muy mermado, al ser valorado (sin ningún apoyo crítico) únicamente el contenido del mismo. Un lenguaje más que asequible, por último (apoyado en ocasiones en algunas breves notas), y el siempre atrayente escapismo de la aventura y los mundos exóticos, promueve, sin duda, el éxito del autor.

Podemos afirmar, para concluir, que el boom editorial de los años ochenta, de lo que entonces se llamó «nueva narrativa», tiene un claro reflejo en Italia, si bien el éxito de estos autores está concentrado en gran medida en la producción de uno de ellos, Manuel Vázquez Montalbán, representante de lo que hasta hace poco se consideraba subgénero literario, actualmente objeto de interés crítico y evidente éxito de ventas.

La variedad de editoriales es muy amplia: la que más destaca del conjunto es Feltrinelli, que opta decididamente por nuestra narrativa, si bien hay que recordar que 17 de los volúmenes son del mismo autor, de Vázquez Montalbán; le siguen en número Frassinelli (adalid con la anterior del «fenómeno Montalbán»), Club degli Editori, y más rezagadas, Sellerio, Sperling \& Kupfer y Sperling Paperback (éstas dos últimas dedicadas a Vázquez Figueroa). Se trata de firmas grandes y medias, muy divulgativas, que aprovechan el éxito de estos autores. No llegan a desaparecer del horizonte, por otro lado, pequeñas editoriales de cultura que se ocupan de títulos menos conocidos (como Biblioteca del Vascello o Le Mani), pero que contribuyen enormemente a la difusión de nuestra narrativa. Prevalece decididamente el criterio semántico de traducción, excepto en lo que concierne a la novela policiaca, siguiendo una tendencia frecuente en este tipo de género (E. Morillas, 1997). Por otra parte, el número traductores que ha colaborado en estos textos es muy amplio, pero se tiende al traductor profesional, alejado del mundo académico y formado como tal. La elaboración editorial en todos ellos es mínima, lo cual condiciona un bajo porcentaje en cuanto a aparato

${ }^{29}$ Otro motivo de incluir sólo las iniciales podría ser que estas traducciones estén excesivamente corregidas por el corrector de pruebas o por los redactores de la editorial; al ser, prácticamente, un trabajo en colaboración, se evita dar su nombre. 
crítico y ediciones anotadas; es decir, predomina el volumen de tipo divulgativo.

El número de publicaciones aumenta progresivamente a partir de 1975 (año de la muerte de Franco) para ascender, sobre todo, a partir de 1989, año fundamental para nuestros autores. La década más importante es la de los noventa, en consonancia con el impulso recibido por los eventos internacionales de 1992 (las Olimpiadas, la Expo, Madrid como capital europea de la cultura); se alcanza la cumbre en 1994, y, tras una breve depresión en 1997, nos enfrentamos a un panorama alentador: en los últimos tres años del siglo la producción se mantiene muy alta, y ello nos hace adoptar un punto de vista optimista en relación con el futuro. Todos ellos, por otra parte, son traducidos pocos años después de publicarse el original español: mientras más conocido es el autor y más potencialmente atrayente (como sucede con Vázquez Montalbán), menor es esta distancia. Por otra parte, es precisamente con tales autores con quienes el mundo editorial se muestra interesado en rescatar obras algo anteriores que puedan sumarse a sus éxitos.

No hay que olvidar, por último, que a partir de los años setenta la industria editorial se transforma completamente, pasando definitivamente a configurarse como estructura empresarial y comercial. Por eso, en este periodo y en el siguiente, la elección de los autores y sus obras va a estar más que nunca relacionada con motivos de mercado. Y no sólo eso: la propia génesis novelesca tendrá en muchas ocasiones (lo que ha valido más de una crítica a los autores actuales) que ver con las decisiones y motivaciones de las diferentes firmas: es la época de los best-seller, que a veces nacen ya como tales antes de ser escritos. Hay, en resumen, un gran impulso editorial que va a aprovechar cada vez más a los autores españoles más modernos y menos encuadrados en una realidad «española» y susceptibles, por tanto, de entrar en los circuitos habituales de promoción y distribución, independientemente de su nacionalidad ${ }^{30}$. Nos gustaría terminar con las palabras de Jordi Gracia (2001: 196), que confirman lo anteriormente dicho: «la novela o el ensayo de autor español no es leído en Europa porque sea español, sino porque es recomendable leerlo, y sólo accidentalmente, administrativamente, obra de un español. El pintoresquismo o la peculiaridad de un país de maltraer ha dejado de funcionar como estímulo insano del lector europeo porque apenas es ya un aliciente saber que procede de este país».

30 Vid. nota 3. 


\section{REFERENCIAS BIBLIOGRÁFICAS}

Acín, R. (1992). Los dedos de la mano. Zaragoza: Mira.

ArpaiA, B. (2002). «Enrique Vila-Matas. La vertigine del silenzio». Il sole/24 ore, 21 abril, 37.

AzÚA, F. DE (1990). Storia di un idiota narrata da lui stesso, o il contenuto della felicità. Parma: Guanda (tr. Elide Pittarello).

- (1993). Cambio di bandiera. Milano: Garzanti (tr. Stefania Cherchi).

Bonino, G. (1995). «Manuel Vicent, Mediterraneo. Mare interiore». L'Indice dei Libri del Mese 9, 34.

Biondi, C. (1996). «Manuel Vázquez Montalbán, Le terme». L’Indice dei Libri del Mese 9, 18.

DíEz, L. M. (1996). I mali minori. Genova: Il Melangolo (ed. y tr. Maria Vittoria Calvi).

Gala, A. (1995). La passione turca. Milano: Rizzoli (tr. Angelo Morino).

GARCíA RodríGuEZ, C. (2003a). Las traducciones italianas de la poesía española del siglo XX (1975-2000). Madrid: UNED (Prólogo de José Romera Castillo).

- (2003b). Il teatro spagnolo del Novecento sulle scene italiane. Firenze: Alinea (Parte de su tesis de doctorado, dirigida por J. Romera Castillo).

GraCiA, J. (2001). Hijos de la razón. Contraluces de la libertad en las letras españolas de la democracia. Barcelona: Edhasa.

JimÉnEZ LozAno, J. (1993). Sara di Ur. Roma: Biblioteca del Vascello (ed. y tr. Danilo Manera).

LANDERO, L. (1991). Giochi tardivi. Milano: Feltrinelli (tr. Gianni Guadalupi).

MANERA, D. (1991). «Barrio delle maraviglie. Scrittori spagnoli d'oggi». Linea d'Ombra 56, 28-31.

MARÍAS, J. (1996). Un cuore così bianco. Roma: Donzelli (tr. Bianca Lazzaro).

- (1998a). Domani nella battaglia pensa a me. Torino: Einaudi (tr. Glauco Felici).

- (1998b). Domani nella battaglia pensa a me. Milano: Club degli editori (tr. Glauco Felici). 
- (1999a). Un cuore cosi bianco. Torino: Einaudi (tr. Paola Tomasinelli).

- (1999b). Tutte le anime. Torino: Einaudi (tr. Glauco Felici).

- (1999c). Tutte le anime. Milano: Club degli editori (tr. Glauco Felici).

- (2000a). L'uomo sentimentale. Torino: Einaudi (tr. Glauco Felici).

- (2000b). Nera schiena del tempo. Torino: Einaudi (tr. Glauco Felici).

MendozA, E. (1987). La città dei prodigi. Milano: Longanesi (tr. Claudio M. Valentinetti).

- (1990). Il mistero della cripta stregata. Milano: Feltrinelli (tr. Gianni Guadalupi).

- (1991). L'isola inaudita. Milano: Feltrinelli (tr. Olivo Bin).

- (1992). Nessuna notizia di Gurb. Milano: Feltrinelli (tr. Gianni Guadalupi).

— (1994). L'anno del diluvio. Recco [Ge]: Le Mani (tr. Fiorenzo Toso).

- (1995). La verità sul caso Savolta. Milano: Feltrinelli (tr. Gianni Guadalupi).

Millás, J. J. (1994). Il disordine del tuo nome. Napoli: Cronopio (ed. y tr. Maria Rosaria Alfani).

Morillas, E. (1997). «Género, estilo y traducción: Beltenebros, de A. Muñoz Molina, en italiano». En E. Morillas y J. P. Arias (eds.), El papel del traductor, 387-398. Salamanca: Ediciones Colegio de España.

Newmark, P. (1995). Manual de traducción. Madrid: Cátedra (tr. Virgilio Moya).

Nicola, M. (1999). «Javier Marías, Tutte le anime». L'Indice dei Libri del Mese 5, 19.

PeÑate Rivero, J. (1997). «El superventas en el marco de la industria editorial. Un estudio empírico de las listas de éxitos». En J. M. López de Abiada y J. Peñate Rivero (eds.), Éxito de ventas y calidad literaria (Incursiones en las teorías y prácticas del best-seller), 53-94. Madrid: Verbum.

PéreZ Vicente, N. (2001). «Cuentistas españolas de los noventa en Italia: las traducciones». En J. Romera Castillo y F. Gutiérrez Carbajo (eds.), El cuento en la década de los noventa, 167-176. Madrid: Visor Libros. 
- (2002). «Narrativa española novecentista y vanguardista publicada en Italia desde 1975: las traducciones». En F. López Criado (ed.), Wenceslao Fernández Flórez: Evasión y Compromiso en la primera mitad del siglo XX, 689-696. La Coruña: Ayuntamiento.

- (2003). «Panorama de la narrativa española del siglo Xx publicada en Italia: algunas conclusiones». En AA.VV., Tradurre dallo spagnolo, 35-45. Milano: LED.

- (2005). «Presencia de las narradoras españolas del siglo XX en Italia: las traducciones». Trans 9, 115-129.

Pittarello, E. (1995). «Le finzioni dell'ombra». En C. Prestigiacomo y M. C. Ruta (eds.), La cultura spagnola degli anni ottanta, 51-63. Palermo: Flaccovio.

Pombo, Á. (1987). L'eroe delle mansarde di Mansard. Milano: Garzanti (tr. Mario Faustinelli).

- (2000). Dove le donne. Torino: Einaudi (tr. Glauco Felici).

PORTO BuCCIARELLI, L. B. (1990). «Letteratura spagnola dai classici ai contemporanei, un variegato percorso». L'Informazione Bibliografica 2, 225-233.

SAMPEDRo, J. L. (1997). Il sorriso etrusco. Milano: Il Saggiatore (tr. Gina Maneri).

- (1999). Il sorriso etrusco. Milano: Tropea (tr. Gina Maneri).

Scorpioni Coggiola, V. (1995). Tempo di silenzio e tempo di parola. Incursioni nel romanzo spagnolo dalla fine della guerra civile ai primi anni '90. Torino: Il Segnalibro.

Tomeo, J. (1989). Amato mostro. Milano: Mondadori (tr. Vittoria Martinetto y Angelo Morino).

- (1991). Il castello della lettera cifrata. Torino: Bollati Boringhieri (tr. Teodora Del Río).

- (1992). I nemici. Torino: Bollati Boringhieri (tr. Anna Baggiani Cases).

- (1993). Il maggiordomo miope. Torino: Bollati Boringhieri (tr. Anna Baggiani Cases).

- (1998). Problemi oculari. Genova: Il Melangolo (ed. y tr. Luigi Dapelo).

- (1999). Il delitto del cinema Oriente. Firenze: Passigli (tr. Barbara Bertoni). 
- (2000). Dialogo in Re maggiore. Firenze: Passigli (tr. Barbara Bertoni).

VÁzQuez FigueroA, A. (1978). Ashanti: la via degli schiavi. Milano: Sperling \& Kupfer (tr. Stefano Bossi).

- (1979a). Ashanti: la via degli schiavi. Milano: Club degli Editori (tr. Stefano Bossi).

- (1979b). Manaos. Milano: Sperling \& Kupfer (tr. Stefano Bossi).

- (1979c). Panama Panama. Milano: Sperling \& Kupfer (tr. Stefano Bossi).

- (1980a). Panama Panama. Milano: Club degli editori (tr. Stefano Bossi).

— (1980b). Manaos. Milano: Club degli Editori (tr. Stefano Bossi).

- (1981). Come un cane rabbioso. Torino: SEI (tr. Laura Tam).

— (1983). Tuareg. Milano: Sperling \& Kupfer (tr. Stefano Bossi).

- (1985a). Il re delle iguane. Sperling \& Kupfer (tr. Stefano Bossi).

- (1985b). Il re delle iguane. Milano: Club degli editori (tr. Stefano Bossi).

— (1986a). La ragazza del mare. Milano: Sperling \& Kupfer (tr. M. A).

- (1986b). La ragazza del mare. Milano: Club degli editori (tr. M. A.).

- (1991). Ashanti: la via degli schiavi. Milano: Sperling Paperback (tr. Stefano Bossi).

— (1992). Manaos. Milano: Sperling Paperback (tr. Stefano Bossi).

— (1994). Tuareg. Milano: Sperling Paperback (tr. Stefano Bossi).

— (1998). Yàiza. Milano: Sperling \& Kupfer (tr. M. A).

— (1999). Il fiume nero dei diamanti. Milano: Sperling \& Kupfer (tr. M. A.).

VÁzQuez Montalbán, M. (1980). Manifesto subnormale. Catania: Pellicano libri (tr. Beniamino Vignola).

- (1982). Un delitto per Pepe Carvalho. Roma: Editori Riuniti (tr. Sonia Piloto di Castri).

- (1984). Assassinio al Comitato Centrale. Palermo: Sellerio (tr. Lucrezia Panunzio Cipriani).

- (1990a). Quartetto. Milano: Marcos y Marcos (tr. Hado Lyria).

— (1990b). Gli uccelli di Bangkok. Milano: Feltrinelli (tr. Sandro Ossola). 
- (1990c). Il pianista. Palermo: Sellerio (tr. e note Hado Lyria).

- (1991a). Il centravanti é stato assassinato verso sera. Milano: Feltrinelli (tr. Hado Lyria).

— (1991b). Galíndez. Milano: Frassinelli (tr. Hado Lyria).

— (1991c). Tatuaggio. Milano: Feltrinelli (tr. Hado Lyria).

- (1992a). Ricette immorali. Milano: Feltrinelli (tr. Hado Lyria; ed. Ferdinando Tempesti; intr. Roberto Freak Antoni).

- (1992b). Il labirinto greco. Milano: Feltrinelli (tr. Hado Lyria).

- (1993a). Gli allegri ragazzi di Atzavara. Milano: Frassinelli (tr. Hado Lyria).

— (1993b). Io, Franco. Milano: Frassinelli (tr. Hado Lyria).

— (1993c). La solitudine del manager. Milano: Feltrinelli (tr. Hado Lyria).

- (1994a). Dallo spillo all' elefante. Milano: Frassinelli (tr. Hado Lyria).

- (1994b). I mari del sud. Milano: Feltrinelli (tr. Hado Lyria).

- (1994c). Le ricette di Pepe Carvalho. Milano: Feltrinelli (tr. Hado Lyria).

- (1995a). La rossa di Alessandria. Milano: Feltrinelli (tr. Hado Lyria).

- (1995b). Lo strangolatore. Milano: Frassinelli (tr. Hado Lyria).

— (1995c). Lo strangolatore. Milano: Club degli Editori (tr. Hado Lyria).

- (1996a). Quartetto. Bussolengo [Ve]: Demetra (tr. Hado Lyria).

— (1996b). Le terme. Milano: Feltrinelli (tr. Hado Lyria).

- (1996c). La rossa di Alessandria. Milano: Club degli Editori (tr. Hado Lyria).

- (1997). Il fratellino. Milano: Feltrinelli (tr. Hado Lyria).

- (1998a). O Cesare o nulla. Milano: Frassinelli (tr. Hado Lyria).

— (1998b). Il premio. Milano: Feltrinelli (tr. Hado Lyria).

- (1999a). Quintetto di Buenos Aires. Milano: Feltrinelli (tr. Hado Lyria).

— (1999b). Storie di fantasmi. Milano: Feltrinelli (tr. Hado Lyria).

— (1999c). Il premio. Milano: Club degli Editori (tr. Hado Lyria). 
- (2000a). Riflessioni di Robinson davanti a centoventi baccalà. Milano: Frassinelli (tr. Hado Lyria).

- (2000b). L'uomo della mia vita. Milano: Feltrinelli (tr. Hado Lyria).

Vicent, M. (1995). Mediterraneo, mare interiore. Milano: Feltrinelli Traveller (tr. Pino Cacucci e Gloria Corica).

Vila-Matas, E. (1989). Storia abbreviata della letteratura portatile. Palermo: Sellerio (tr. Lucrezia Panunzio Cipriani).

- (1994). Suicidi esemplari. Palermo: Sellerio (tr. Lucrezia Panunzio Cipriani).

ZÚÑIGA, J. E. (1994a). La terra sarà un paradiso. Roma: Biblioteca del Vascello (ed. Danilo Manera; tr. y epíl. Tonina Paba).

- (1994b). L'anello di Puskin. Roma: Biblioteca del Vascello (ed. Danilo Manera; tr. Laura Giani, Danilo Manera, Margherita Guffanti, Anna Maria Zerla, Roberta Bellintani, Barbara Malanca y Sara Scrinzi). 